\title{
Prognostic significance of paraproteinaemia in rheumatoid arthritis
}

\author{
Clive Kelly, Gillian Baird, Helen Foster, Harold Hosker, Ian Griffiths
}

\begin{abstract}
The incidence of lymphoma and myeloma is increased in rheumatoid arthritis (RA), though the reasons for this are incompletely understood. The predictive value of monoclonal gammopathy in RA for the later development of lymphoproliferative malignancy was assessed. A serum paraprotein was discovered in 23 patients ( 14 female) with a median age of 58 years and a median duration of RA of six years. The paraprotein was quantified and further investigations, including a bone marrow examination and skeletal survey, were undertaken to study the possible development of a lymphoproliferative disorder. Secondary Sjögren's syndrome was noted in 12 patients.

The paraprotein was monoclonal in 21 patients and was classed as IgG (12 cases), IgA (six cases), or IgM (three cases). Biclonal paraproteins were seen in two patients. Free light chains were found in the urine of eight patients. Bone marrow abnormalities were found in 13 subjects. The bone marrow was abnormal in four of six patients with an IgA paraprotein, in two of three with an IgM paraprotein but in only five of 12 patients with an IgG paraprotein. Patients were followed up for a median of four years, and five patients with an abnormal bone marrow developed myeloma, of whom three had an IgA $\lambda$ paraprotein. Two other patients developed nonHodgkin's lymphoma. Extra-articular bone erosions were seen in four patients, three of whom developed myeloma, but the presence of urinary free light chains was not specific for the development of malignancy. Paraproteinaemia in RA indicates monoclonal $B$ cell proliferation and carries a high risk of malignant transformation.
\end{abstract}

Departments of

Rheumatology and

Haematology,

Royal Victoria Infirmary,

Victoria Road,

Newcastle upon Tyne,

Tyne and Wear

C Kelly

G Baird

H Foster

H Hosker

I Griffiths

Correspondence to:

Dr Clive Kelly,

Department of Rheumatology

Royal Victoria Infirmary,

Victoria Road

Newcastle upon Tyne,

Tyne and Wear.

Accepted for publication

28 June 1990
A relation between rheumatoid arthritis (RA) and myeloma was proposed by Galli and Chiti in $1955^{1}$ and supported by Goldenberg et al. ${ }^{2}$ Firm evidence that RA is associated with an excess risk of both myeloma and lymphoma was first obtained from a large population study in Finland in $1982 .{ }^{3}$ Confirmation of the increased risks of myeloma and lymphoma in RA have recently been obtained from smaller studies with clearer diagnostic criteria. ${ }^{45}$ There is evidence that the risk of these malignancies relates to the duration of $R A,{ }^{6}$ but there is little published information on other risk factors. In particular, the possible relevance of monoclonal gammopathy to malignancy in rheumatic disease has only recently been discussed. ${ }^{78}$ In this longitudinal study of patients with paraproteinaemia we attempted to evaluate the risk of lymphoproliferative malignancy and relate it to a variety of easily measured parameters.

\section{Patients and methods}

We identified 23 patients with $\mathrm{RA}^{9}$ and a serum monoclonal or biclonal paraprotein who had attended a district rheumatology unit serving a population of 800000 over an eight year period. Their case notes were obtained and the following demographic data recorded: age and sex; date of diagnosis of RA; date of detection of paraprotein by densitometry and concentration of other immunoglobulins at that time. The presence of free light chains in the urine was noted. IgM rheumatoid factor titres and the presence or absence of secondary Sjögren's syndrome were also recorded from the case notes. Sjögren's syndrome was considered present when sicca symptoms were supported by the finding of an abnormal Schirmer test with less than $10 \mathrm{~mm}$ wetting bilaterally over five minutes. Skeletal surveys were carried out on all patients, and in those found to have extraarticular erosions an isotope bone scan was undertaken.

All patients had undergone bone marrow aspiration and trephination, and samples were independently reviewed by one of us (GB) without knowledge of the report previously issued by the attending haematologist. The following details were recorded: overall cellularity, plasma cell morphology, percentage, and distribution on trephination. When the reports differed significantly a second observer was asked to examine the slides without reference to previous reports.

Although most patients had been followed up on a regular basis in the rheumatology department, all patients were recalled to the outpatient clinic and sequential changes in paraprotein and immunoglobulin concentrations were recorded from haematology record sheets. Determinations of autoantibody titres and immunoglobulin concentrations were specifically repeated at this visit. Bone marrow examination was repeated only in patients with a strong clinical indication. All treatment received since detection of the paraprotein was recorded.

Statistical comparisons between those paraprotein concentrations found initially and those found at follow up were made with Student's unpaired $t$ test, whereas the Wilcoxon rank test was used to compare paraprotein concentrations between subgroups. 
Table 1 Demographic details of 23 patients with rheumatoid arthritis (RA) and a serum paraprotein at follow up

\begin{tabular}{llrl}
\hline $\begin{array}{l}\text { Patient } \\
\text { No }\end{array}$ & $\begin{array}{l}\text { Age at } \\
\text { onset of } R A \\
\text { (years) }\end{array}$ & $\begin{array}{l}\text { Duration of } \\
R A \text { (years) }\end{array}$ & $\begin{array}{l}\text { Duration of } \\
\text { paraprotein } \\
\text { (years) }\end{array}$ \\
\hline 1 & 42 & 8 & 8 \\
2 & 52 & 16 & 2 \\
3 & 59 & 5 & 5 \\
4 & 49 & 1 & 1 \\
5 & 62 & 5 & 4 \\
6 & 61 & 13 & 7 \\
7 & 43 & 35 & 5 \\
8 & 64 & 7 & 4 \\
9 & 71 & 5 & 5 \\
10 & 58 & 10 & 7 \\
11 & 29 & 35 & 2 \\
12 & 52 & 7 & 6 \\
13 & 43 & 14 & 4 \\
14 & 71 & 5 & 1 \\
15 & 61 & 5 & 1 \\
16 & 43 & 19 & 9 \\
17 & 56 & 9 & 7 \\
18 & 18 & 38 & 1 \\
19 & 42 & 16 & 2 \\
20 & 56 & 21 & 3 \\
21 & 52 & 3 & 3 \\
22 & 48 & 7 & 5 \\
23 & 50 & 18 & 1 \\
& & & 4 (1-9) \\
Median (range) & $52(18-71)$ & 10 (1-38) \\
\hline
\end{tabular}

\section{Results}

Fourteen of our 23 patients were female, giving a female:male ratio of about $1 \cdot 5: 1$. The median age of the group at discovery of the paraprotein was 58 years (range 41 to 75 ) and the median duration of RA at this time was six years (range 0 to 37). Diagnosis of RA and discovery of paraproteinaemia were synchronous in five patients, but in none was the paraprotein found before the onset of joint symptoms. Table 1 provides individual demographic details, including the duration of follow up.

\section{PARAPROTEIN}

Initial assessment showed the paraprotein was monoclonal in 21 patients and biclonal in two. It

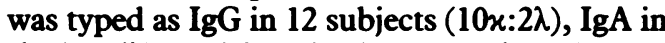
six $(1 x: 5 \lambda)$, and IgM in three $(2 x: 1 \lambda)$. The two patients with a biclonal paraprotein both had $\operatorname{IgA} \lambda$ bands, together with an IgG $x$ in one case and an IgM $x$ in the other. The median concentration of paraprotein was $9.0 \mathrm{~g} / \mathrm{l}$ (range $2 \cdot 3-33 \cdot 0$ ) and did not differ significantly between immunoglobulin subclasses. Immune paresis with depression of the other immunoglobulin concentrations was seen at this stage in two patients, whereas a polyclonal increase in immunoglobulins was evident in four subjects with secondary Sjögren's syndrome (table 2).

Free light chains were found in the urine of eight patients. Four had free $x$ chains, three had $\lambda$ chains, while one patient with a biclonal paraprotein had both. Twenty patients were

Table 2 Initial paraprotein concentrations in 23 patients with rheumatoid arthritis, grouped by paraprotein subclass

\begin{tabular}{llll}
\hline Paraprotein & $x: \lambda$ Ratio & $\begin{array}{l}\text { Paraprotein median } \\
\text { concentration } \\
(\text { range })(g / l)\end{array}$ & Other immunoglobulins* \\
\hline IgG $(n=12)$ & $10: 2$ & $10 \cdot 8(2 \cdot 3-33 \cdot 0)$ & Normal $8 ; \uparrow 2 ; \downarrow 2$. \\
$\operatorname{IgA}(n=6)$ & $1: 5$ & $7 \cdot 6(3 \cdot 4-12 \cdot 4)$ & Normal $3 ; \uparrow 0 ; \downarrow 3$. \\
IgM $(n=3)$ & $2: 1$ & $8 \cdot 2(3 \cdot 4-18 \cdot 0)$ & Normal $2 ; \uparrow 1 ; \downarrow 0$. \\
Biclonal $(n=2)$ & & & Normal $1 ; \uparrow 1 ; \downarrow 0$. \\
\hline
\end{tabular}

$\uparrow \uparrow$ =increased; $\downarrow$ = decreased. seropositive for IgM rheumatoid factor and three patients were seronegative at the time the paraprotein was detected. Titres of rheumatoid factor ranged from $1 / 160$ to $1 / 1280$ and 12 patients had secondary Sjögren's syndrome. Erosive joint disease was present in all seropositive patients and two seronegative patients. Extra-articular erosions were seen in four patients on the skeletal survey, of whom three were seropositive. Bony lysis was present in the humeri in three of these patients and in the femora in the remaining patient. Isotope bone scans were positive in each of these four patients.

\section{BONE MARROW}

Bone marrow examination showed significant abnormalities in 13 patients. These usually included an increase in overall cellularity with an expansion of the plasma cell population. More specific features included the presence of binucleate, blastic, or lymphoplasmacytoid plasma cells. Abnormal plasma cell distribution was also seen with focal and paratrabecular aggregates. In one patient direct immunofluorescence was used to confirm the monoclonality of plasma cells. The independent review was significantly different from the initial report in three patients and thus the opinion of the second observer was sought. This concurred with the findings of the independent reviewer in each case.

An abnormal bone marrow was found in four of six patients with an IgA paraprotein and in two of three subjects with an IgM paraprotein. Five of 12 patients with an IgG paraprotein and both the patients with a biclonal paraprotein were also found to have an abnormal marrow. The risk of an abnormal bone marrow was no greater among the patients with secondary Sjögren's syndrome nor among those with urinary free light chain excretion. Furthermore, in only two of the five patients with both secondary Sjögren's syndrome and free light chain excretion was the bone marrow found to be abnormal.

\section{FOLLOW UP}

All 23 patients were reviewed at a median of four years (range 1-9) after the initial report of their paraproteinaemia. Table 3 shows that median concentrations of paraprotein had increased from their initial values in all immunoglobulin subclasses, though this increase was significant only for IgA paraprotein $(p<0.05)$. Overall, the median paraprotein concentration rose slightly to $10.9 \mathrm{~g} / \mathrm{l}$ (range $0-60$ ) but was no longer detectable in two patients with low concentrations of paraprotein initially. Paraprotein concentrations were stable in 17 patients and had risen by more than $5 \mathrm{~g} / \mathrm{l}$ in only four subjects. Immune paresis had developed in two of these patients during follow up. Titres of rheumatoid factor did not alter significantly during the follow up period, and none of the three seronegative patients became seropositive.

Five of the 23 patients reviewed had developed myeloma,${ }^{10}$ of whom three had an IgA $\lambda$ mono- 
Table 3 Follow up data in 23 patients with rheumatoid arthritis and a paraprotein, grouped by paraprotein subclass

\begin{tabular}{lllll}
\hline Paraprotein & $\begin{array}{l}\text { Paraprotein median } \\
\text { concentration } \\
\text { (range) }(\mathrm{g} / \mathrm{l})\end{array}$ & $\begin{array}{l}\text { Abnormal marmow } \\
\text { No }(\%)\end{array}$ & $\begin{array}{l}\text { Myeloma } \\
\text { No (\%) }\end{array}$ & $\begin{array}{l}\text { Lymphoma } \\
\text { No (\%) }\end{array}$ \\
\hline IgG $(\mathrm{n}=12)$ & $11 \cdot 3(0-60 \cdot 0)$ & $5(42)$ & $2(17)$ & $1(8)$ \\
$\operatorname{IgA}(\mathrm{n}=6)$ & $12 \cdot 4(0-16 \cdot 6)$ & $4(67)$ & $3(50)$ & 0 \\
$\operatorname{IgM}(\mathrm{n}=3)$ & $10 \cdot 0(5 \cdot 2-18 \cdot 4)$ & $2(67)$ & 0 & $1(33)$ \\
Biclonal $(\mathrm{n}=2)$ & & $2(100)$ & 0 & 0 \\
\hline
\end{tabular}

clonal paraprotein. Two further patients had histologically proved non-Hodgkin's lymphoma at review. Five of these seven patients were male. No patient developed both these malignancies, and no other neoplasms developed during the follow up period. Three of four patients with immune paresis developed myeloma, whereas none of the patients with a polyclonal rise in immunoglobulins developed malignancy. Table 3 shows the relation between outcome and paraprotein type. Bone marrow examinations were repeated in only four patients. This was performed to assess possible progression towards lymphoproliferative change in three patients with high concentrations of paraprotein, and showed features of myeloma in one of these. In one patient with previously established myeloma a repeat bone marrow examination was done to assess the response to treatment.

Both patients with non-Hodgkin's lymphoma had secondary Sjögren's syndrome, which was present in only two of the patients with myeloma. Two patients with myeloma but neither of those with non-Hodgkin's lymphoma had free urinary light chain excretion. Three of four patients with extra-articular erosions developed myeloma. The duration of RA was at least 12 years in all patients who developed malignancy, with the one exception having developed myeloma six years after the onset of RA.

\section{TREATMENT}

All patients had received non-steroidal antiinflammatory drugs at some stage. Four patients had been given second line treatment with intramuscular gold and treatment with penicillamine had been started in one. Three patients had received oral steroids. No patient had been treated with cytotoxic drugs for RA, though one had received chlorambucil for thrombocytopenic purpura.

Both patients with non-Hodgkin's lymphoma were treated with radiotherapy and one underwent surgery to debulk tumour mass. One patient died, but the other was well at follow up with no evidence of recurrent tumour. Three patients with myeloma underwent treatment with cyclical melphalan and prednisolone. One patient remains anaemic and unwell four years after diagnosis, but the other two are presently well one and five years after treatment. A fourth patient is about to start treatment, and treatment has been unnecessary in one virtually asymptomatic woman.

\section{Discussion}

Our study suggests that monoclonal gammopathy in RA carries a high risk for the development of later lymphoproliferative malignancy. Mortality is increased in patients with $\mathrm{RA},{ }^{11-13}$ but there has been only limited evidence that lymphoproliferative disorders contribute significantly to this excess. ${ }^{14}$ Indeed, in a survey of patients with lymphoproliferative malignancy Miller was unable to show an excess of patients with RA, ${ }^{16}$ and Owen et al could find no increase in neoplasia of the lymphoreticular system in a review of almost 200 patients with RA. ${ }^{17}$ Larger surveys of patients with reticuloses $^{18}$ or RA, ${ }^{19}$ however, have shown firm evidence of an association between lymphoma and RA. This is consistent with the situation in Sjögren's syndrome where the relative risk of developing non-Hodgkin's lymphoma has been estimated to be as high as $44 .{ }^{20}$

Less attention has been paid to a possible association between RA and myeloma. In two series reported 20 years ago, however, six cases of myeloma occurred in a group of 16 patients with RA and paraproteinaemia, ${ }^{21}$ and a further five patients with RA and a paraprotein developed myeloma. ${ }^{22}$ Although prognostic factors were not discussed, IgA and IgM paraproteins occurred almost as often as IgG paraproteins in these patients. Paraproteinaemia occurs in about $1 \%$ of patients under the age of 60 and thereafter its incidence rises sharply with age. ${ }^{23}$ Estimates for the risk of evolution into a malignant disorder vary widely from $0.5 \%$ in patients with a low concentration of a stable paraprotein ${ }^{24}$ to as many as $28 \%$ of elderly patients over a 10 year period. 25

In 296 patients with monoclonal gammopathy Waldenström found three patients with RA. ${ }^{26} \mathrm{~A}$ further three patients with RA were reported in a series of 150 cases of paraproteinaemia, ${ }^{27}$ giving an overall prevalence of $1 \cdot 3 \%$. In two large series a total of eight cases of monoclonal gammopathy were found among 640 patients with seropositive $R A,{ }^{21}$ giving a prevalence of $1 \cdot 25 \%$. As both RA and paraproteinaemia occur in about $1 \%$ of the population the association between the two conditions at best seems to be slight. The significance of monoclonal gammopathy in RA differs from that in the normal population, however. We suggest that the development of lymphoproliferative disorders in some patients with RA may be predicted by the presence of monoclonal paraprotein which, although not found in gross excess in a rheumatoid population, seems to carry a much higher risk of malignant transformation in patients with RA than in the population at large. The increased risk of lymphoma and myeloma in RA may be due to chronic immune stimulation and the associated B lymphocyte activation, ${ }^{28}$ and rheumatoid lymph nodes are known to be a major site of this B cell driven activity. ${ }^{29}$

The class of paraprotein present may be of some prognostic value. Previous studies have reported more IgA and less IgG paraproteins in patients with RA than found in the general population. ${ }^{21} 2230$ Our findings are similar, and suggest that the risk of myeloma may be greater in those patients with a monoclonal $\operatorname{IgA} \lambda$ 
paraprotein. The $x: \lambda$ light chain ratio in our patients with an IgA paraprotein was 0.2 , which contrasts with the ratio of 1.3 reported in a large series of patients with monoclonal gammopathy without $R A .{ }^{30}$ The reported $x: \lambda$ ratios in the other paraprotein types were similar to those found in our study. Other well recognised factors, such as a high concentration of paraprotein and the presence of immune paresis, were also predictive of malignancy in our patients.

The presence of urinary free light chains has been proposed as a marker of malignancy in patients with primary Sjögren's syndrome. ${ }^{31} 32$ We were unable to show a relation between free light chain excretion and malignant transformation in our patients with secondary Sjögren's syndrome. Both our patients who developed lymphoma had associated secondary Sjögren's syndrome, however, and the increased risk of lymphoma in Sjögren's syndrome is unaffected by the presence of associated connective tissue disease. ${ }^{20}$ Amyloidosis may also complicate longstanding RA and can be associated with the production of paraprotein. No evidence of amyloid was found in any of our patients, though it was not actively sought unless felt to be of potential clinical relevance.

Bone marrow examinations were abnormal in over half our patients. Abnormality can be difficult to define in patients with $R A$ as a reactive plasmacytosis is commonly associated with active joint disease, but was based more on atypical morphology and distribution of plasma cells than on their numbers alone. Certain features, such as focal aggregation or paratrabecular distribution of plasma cells, suggest the development of malignancy, ${ }^{33}$ but strict criteria for the diagnosis of myeloma were met in only five patients during the follow up period. ${ }^{10}$ The bone marrow was abnormal in both patients who later developed lymphoma. Six patients with excessive or abnormal plasma cells have not developed lymphoproliferative disease, and this group includes both patients with biclonal gammopathy. One patient with an IgG paraprotein has now developed immune paresis, however, and another with an IgM paraprotein has findings suggestive of early Waldenström's macroglobulinaemia, so clearly this borderline group remains a cause for concern and later progression to lymphoproliferative malignancy remains a strong possibility.

Only eight patients had received treatment with second line agents or oral steroids, and there is no evidence that these drugs predispose to malignancy. One other patient had received cytotoxic drugs. There is growing concern that the use of such agents in patients with severe RA may increase the risk of later lymphoma, ${ }^{34}$ though this patient has remained well. A single death has occurred among our seven patients with lymphoproliferative disease, but it is too early to speculate on the long term prognosis.

Our study offers evidence that the development of paraproteinaemia in patients with RA is related more to disease severity than to duration. The median age of our group when a paraprotein was first detected was 58 years and the average duration of RA at this stage was six years, while most of our patients had erosive disease and were seropositive. We suggest that paraproteinaemia occurring in patients with RA should be promptly investigated with a skeletal survey and a bone marrow examination. Elderly men with severe disease are most at risk of developing a lymphoproliferative disorder, and the presence of an $\operatorname{IgA\lambda }$ paraprotein may indicate a special risk of myeloma. All patients with morphological abnormalities on bone marrow examination should be followed up six months later with paraprotein measurement and immunoglobulin estimation. A rise of $5 \mathrm{~g} / 1$ in paraprotein concentration over one year or the development of immune paresis should prompt reinvestigation as the response to treatment of lymphoproliferative disease seems to be at least as good as that obtained in patients without RA.

The authors gratefully acknowledge all consultants who made available material included in this paper. We thank especially $\mathrm{Dr}$ Peter Saunders for his invaluable comments and critique.

1 Galli T, Chiti E. Rheumatoid arthritis and plasmacytomatosis. Ann Rheum Dis 1955; 14: 271-7.

2 Goldenberg G J, Paraskevas F, Israels L G. The association of rheumatoid arthritis with plasma cell and lymphocytic neoplasms. Arthritis Rheum 1969; 12: 569-77.

3 Isomaki H A, Hakulinen T, Joutsenlahti U. Excess risk of lymphoma, leukaemia and myeloma in patients with rheumatoid arthritis. Ann Rheum Dis 1982; 41 (suppl): 34-8.

4 Katusic S, Beard C M, Kurland L T, Weiss J W, Bergstralh E. Occurrence of malignant neoplasms in the Rochester, E. Occurrence of malignant neoplasms in the Rochester,
Minnesota rheumatoid arthritis cohort. Am $\mathcal{F}$ Med 1985; 78: $50-5$.

5 Banks P M, Witrak G A, Conn D L. Lymphoid neoplasia developing after connective tissue disease. Mayo Clin Proc 1979; 54: 104-8.

6 Symmons D P M, Ahern M, Bacon P A, et al. Lymphoproliferative malignancies in rheumatoid arthritis. Ann Rheum Dis 1984; 43: 132-3.

7 Hurst N P, Smith W, Henderson D R. IgG kappa paraproteinaemia and arthritis. $B r \mathcal{F}$ Rheumatol 1987; 26 : 142-6.

8 Pruzanski W. Rheumatological disorders and monoclonal gammopathy-a new syndrome? $B r \mathcal{F}$ Rheumatol 1987; 26: g06-8.

9 Arnett F C, Edworthy F M, Bloch D A, et al. The American Rheumatism Association 1987 revised criteria for the Rheumatism Association 1987 revised criteria for the
classification of rheumatoid arthritis. Arthritis Rheum 1988; classification

10 Durie B G M, Salmon S E. Multiple myeloma, macroglobulinaemia and monoclonal gammopathies. In: Hoffbrand $A \mathrm{~V}$, Brain M D, Hirson J, eds. Recent advances in haematology. Vol 2. Edinburgh: Churchill Livingstone, 1977: 133-4.

11 Mutru O, Laakso $M$, Isomaki $H$, Koota $K$. Ten year mortality and causes of death in patients with rheumatoid arthritis. BMF 1985; 290: 1797-9.

12 Vandenbroucke J P V, Hazevoet H M, Cats A. Survival and cause of death in rheumatoid arthritis. $\mathcal{F}$ Rheumatol 1984; 11: $158-61$.

13 Mitchell D M, Spitz P W, Young D Y, Bloch D A, McShane D J, Fries J F. Survival, prognosis and causes of death in D J, Fries J F. Survival, prognosis and causes of death in
rheumatoid arthritis. Arthritis Rheum 1986; 29: 706-14.

14 Prior P, Symmons D P M, Scott D L, Brown R, Hawkins C F. Cause of death in rheumatoid arthritis. $\mathrm{Br} f$ Rheumatol 1984; 23: 92-9.

15 Monson R R, Hall A P. Mortality among arthritis. foumal of Chronic Diseases 1976; 29: 459-67.

16 Miller D G. The association of immune disease and malignan lymphoma. Ann Intern Med 1967; 66: 507-21.

17 Owen D T, Waller M, Toone E. Rheumatoid arthritis and malignancy. Arthritis Rheum 1967; 10: 302-3.

18 Lea $A$ J. An association between the rheumatic diseases and the reticuloses. Ann Rheum Dis 1964; 23: 480-4.

19 Prior P, Symmons D P M, Hawkins C F, Scott D L, Brown R. Cancer morbidity in rheumatoid arthritis. Ann Rheum Dis 1984; 43: 128-31.

20 Talal N, Bunim J J. The development of malignant lymphoma in the course of Siogren's syndrome. Am $\mathcal{J}$ Med 1964; 36: in the cour $529-40$.

21 Zawadzki Z A, Benedek T G. Rheumatoid arthritis, dysproteinemic arthropathy and paraproteinemia. Arthritis Rheum 1969; 12: 555-63.

22 Wegelius O, Skrifvars B, Andersson L. Rheumatoid arthritis terminating in plasmacytoma. Acta Med Scand 1970; 187 133-8. 23 Anonymous. Paraproteinaemia [Editorial]. BMF 1980; 280: 
24 Hobbs J R. Monitoring immunocytoma. Minerva Med 1978; 69: 2499-506.

25 Kyle R A. 'Benign' monoclonal gammopathy-misnomer? JAMA 1984; 251: 1849-54.

26 Waldenström J. Clinical diagnosis and biochemical findings in material of 296 sera with $M$-type marrow $\gamma$ globulins. Acta Med Scand 1961; 170 (suppl 367): 110.

27 Hallen J. Discrete gammaglobulin ( $M$ component) in serum. Acta Med Scand 1966; (suppl 462): 1-127.

28 Symmons D P M. Neoplasia in rheumatoid arthritis. f Rhewmatol 1988; 15: 1319-22.

29 Symmons D P M, Bacon P A, Salmon M, Blann A D. A study of lymph node lymphocytes in rheumatoid arthritis. Adv Exp Med Biol 1985; 186: 1027-32.

30 Malacrida V, Francesco D D, Banfi G, Porta F A, Riches $P$ G. Laboratory investigation of monoclonal gammopathy during 10 years of screening in a general hospital. $f$ Clin Pathol 1987; 40: 793-7.
31 Walters M T, Stevenson F K, Herbert A, Cawley M I D, Smith J L. Urinary monoclonal free light chains in primary Sjögren's syndrome: an aid to the diagnosis of malignant lymphoma. Ann Rherm Dis 1986; 45: 210-9.

32 Moutsopoulos H M, Costello R, Drosos A A, Mavridis A K, Papadopoulos N M. Demonstration and identification of monoclonal proteins in the urine of patients with Sjögren's monoclonal proteins in the urine of patients with
syndrome. Ann Rheum Dis 1985; 44: 109-12.

33 Bartl R, Frisch B, Burkhardt R, Jager K, Pappenberger R, Hoffman-Feyer $G$. Lymphoproliferation in the bone marrow: identification and evolution; classification and staging. $\mathcal{F}$ Clin Pathol 1987; 87: 342-55.

34 Baltus J A M, Boersma J W, Hartman A P, Vandenbroucke $J$ P. The occurrence of malignancies in patients with rheumatoid arthritis treated with cyclophosphamide: a controlled retrospective follow-up. Ann Rheum Dis 1983; 42: 368-73. 